\title{
Osservatori del paesaggio. Materiali per la definizione di un percorso comune e operativo
}

Carlo A. Gemignani

\section{(2) OpenEdition \\ 1 Journals}

Edizione digitale

URL: http://journals.openedition.org/edl/519

DOI: $10.4000 /$ edl. 519

ISSN: 2296-5084

\section{Editore}

Université de Lausanne

\section{Edizione cartacea}

Data di pubblicazione: 15 mai 2013

Paginazione: 345-358

ISBN: 978-2-940331-32-1

ISSN: 0014-2026

Notizia bibliografica digitale

Carlo A. Gemignani, «Osservatori del paesaggio. Materiali per la definizione di un percorso comune e operativo ", Études de lettres [Online], 1-2 | 2013, online dal 15 mai 2016, consultato il 18 décembre 2020. URL : http://journals.openedition.org/edl/519; DOI : https://doi.org/10.4000/edl.519 


\section{OSSERVATORI DEL PAESAGGIO. MATERIALI PER LA DEFINIZIONE DI UN PERCORSO COMUNE E OPERATIVO}

L'examen du contexte régional de la Ligurie et de la Toscane permet à l'auteur de faire le point sur un outil de conservation de la mémoire et de la gestion du paysage: l'Observatoire du paysage. La constitution d'un réseau de ces observatoires sera prochainement réalisée en Ligurie. L'exemple de l'Osservatorio apuano del paesaggio favorisera le débat local sur l'efficacité de ces structures explicitement prévues par la Convention Européenne du Paysage, signée à Florence le 20 octobre 2000.

\section{Osservatori del paesaggio in Liguria: un'occasione mancata}

La successione logica delle parole chiave contenute nel sottotitolo del Laboratorio Internazionale sul Paesaggio di San Biagio della Cima (15 gennaio 2011) - memoria, percezione, rappresentazione, salvaguardia, progettazione territoriale ${ }^{1}$ - rispecchia perfettamente la dimensione progettuale che ritroviamo alla base delle finalità degli Osservatori del paesaggio raccomandati dal Consiglio d'Europa. L'occasione del Laboratorio ha consentito di affrontare il tema attraverso gli interventi del presidente dell'Osservatorio del paesaggio per il Monferrato e l'Astigiano, Marco Devecchi, che ha ricordato un'esperienza italiana "pilota», e di Massimo Quaini, che ha proposto all'Associazione amici di Francesco Biamonti la costituzione di un'analoga struttura per il Ponente ligure.

I. URL: http://www.francescobiamonti.it/eventi/paesaggio_relazione.html (consultato il 31.01.2012). 
La Liguria - intesa come insieme delle amministrazioni locali - se da un lato non manca della consapevolezza di essere una regione speciale dal punto di vista paesaggistico, come del resto dimostra l'intenso impegno nel campo della pianificazione ${ }^{2}$, d'altra parte dei suoi paesaggi mostra di avere privilegiato gli aspetti più immediatamente monetizzabili a sostegno di un turismo che ha comportato un consistente consumo di suolo ${ }^{3}$, proseguito in anni recenti anche attraverso deroghe agli stessi Piani e responsabile, non solo del degrado dello stesso paesaggio, ma anche di una diffusa situazione di dissesto idro-geologico.

Sappiamo come il problema del consumo di suolo sia ormai centrale nel dibattito nazionale ${ }^{4}$ e possa essere considerato come una delle eredità di una radicata e diffusa cultura politica "sviluppista» che ha considerato - e in larga misura considera tuttora - ineluttabile il tramonto del "rurale» 5 . Studi recenti, condotti anche da gruppi di ricercatori genovesi, hanno viceversa messo in luce non solo il legame ineludibile fra agricoltura e qualità del paesaggio, ma anche i meccanismi specifici che legano le singole pratiche alle forme paesistiche che ne scaturiscono ${ }^{6}$.

2. La Regione Liguria è stata la prima a dotarsi di un Piano paesistico, adottato nel 1986 e approvato nel 1990 (delibera del consiglio regionale n.6 del 25 febbraio 1990), ora in fase di revisione. Con la delibera n.18 del 2 agosto 2011, il Consiglio regionale ha inoltre approvato la variante di salvaguardia della fascia costiera.

3. Non si dimentichi che è stata proprio la speculazione edilizia ligure a sollecitare la coniazione del termine "rapallizzazione».

4. Sul tema sta nascendo a ritmo sostenuto una bibliografia specifica. Per dare qualche coordinata di ordine generale mi limito a segnalare la lucida analisi dei meccanismi economico-giuridici alla base del fenomeno compiuta da S. Settis con Paesaggio, Costituzione, cemento e i rapporti annuali del Centro di Ricerche sui Consumi di Suolo (URL: http://www.consumosuolo.org, consultato il 20.12.2012).

5. Una cultura che sembra purtroppo permeare la componente politica di centrosinistra, stabilmente al comando del governo regionale, almeno attenendoci alla produzione di documenti programmatico-normativi in materia. L'unica recente proposta di legge volta a promuovere il recupero di forme di produzione agricola in Liguria, «Nuove disposizioni in materia di utilizzazione agraria dei terreni » $\left(\mathrm{n}^{\circ} 211 / 2011\right.$; URL: http://www.regione.liguria.it/argomenti/media-e-notizie/archivio-comunicati-stampadel-consiglio/archivio-comunicati-stampa-dei-gruppi-consiliari/item/31037-stopallo-spopolamento-dell-entroterra-recuperando-i-terreni-agricoli.html; consultato il 29.03.2012), porta la firma di esponenti politici del centro-destra.

6. Si rimanda a R. Cevasco, Memoria verde, e alla recente tesi di dottorato di C. Vaccarezza, Paesaggi rurali. 
Per rendersi poi conto dell'intreccio di interessi nascosto dietro alle generiche promesse di tutela di un paesaggio ridotto a scenografia e sottoposto invece a continui interventi di erosione della sua dimensione materiale e patrimoniale ${ }^{7}$, basta sfogliare le pagine "virtuali» del blog di Marco Preve ${ }^{8}$, un giornalista impegnato sul fronte della denuncia della nuova speculazione edilizia ligure, o quelle "cartacee" della clamorosa indagine pubblicata quattro anni fa dallo stesso Preve assieme al collega Ferruccio Sansa ${ }^{9}$, recentemente aggiornata e ampliata alla scala nazionale ${ }^{10}$. Agli occhi dello storico, del geografo, del pianificatore, $\mathrm{i}$ materiali forniti da queste forme di giornalismo d'inchiesta forniscono, e forniranno in futuro, fonti interessanti sulla base delle quali si potrà riscrivere la recente storia territoriale ligure.

Gli Osservatori, che dovrebbero costituire uno dei principali «strumenti di decodificazione» del paesaggio, si fondano su una concezione olistica per cui non è la singola emergenza ad essere meritevole di attenzione, ma il paesaggio nella complessità delle relazioni materiali e culturali che lo hanno prodotto in quanto frutto dell'azione storica delle collettività umane nell'insieme del territorio ${ }^{11}$. Sul piano, poi, dell'azione politica, gli Osservatori si distinguono per la fiducia verso la partecipazione e il coinvolgimento dei portatori di interesse locali ${ }^{12}$. In questi due aspetti si possono trovare le potenzialità sia per affrontare le nuove forme di speculazione negli spazi non soggetti a tutela, sia per

7. Sul concetto di Patrimonio territoriale (a sua volta legato a quello di paesaggio) si rimanda agli scritti di A. Magnaghi. Per un rapida sintesi dei suoi fondamenti mi sembra utile segnalare i documenti preparatori al Piano paesaggistico territoriale regionale della Regione Puglia, in particolare le relazioni (2007-2008-2009) a firma dello stesso Magnaghi (URL: http://paesaggio.regione.puglia.it/index.php/area-download.html, consultato il 20.12.2012). Si veda anche P. Baldeschi, Paesaggio e territorio, p. 91-93.

8. URL: http://preve.blogautore.repubblica.it/ (consultato il 31.01.2012).

9. M. Preve, F. Sansa, Il partito del cemento.

Io. A. Garibaldi et al., La Colata.

II. Sulla complessa relazione territorio/paesaggio rimando, oltre che al volume di P. Baldeschi, al Documento preliminare per la commissione epistemologica curato da L. Bonesio e disponibile sul sito della Società dei Territorialisti/e (URL: http://www. societadeiterritorialisti.it/index.php ?option $=$ com_content $\& v i e w=$ article $\&$ id $=197 \&$ Ite mid=103, consultato il 20.12.2012), che inquadra il problema nelle sue linee critiche principali.

I2. P. Baldeschi, Paesaggio e territorio, p. 100-106. 
correggere un approccio ai problemi nelle aree soggette a tutela che non sembra avere dato i frutti attesi.

Tornando alla Liguria è molto interessante, su questo ultimo aspetto, la critica che da anni va portando Sandro Lagomarsini, parroco di Cassego in Val di Vara (SP) che, da un punto di vista privilegiato, ha sempre criticato le politiche vincolistiche in parte responsabili della diminuzione delle attività rurali necessarie alla "manutenzione" dell'ambiente e del paesaggio nelle aree montane ${ }^{13}$.

La necessità della ridiscussione degli attuali sistemi di vincolo è confermata dai dati recentemente raccolti nell'esito editoriale di una ricerca, destinata alla creazione di un Catalogo nazionale dei paesaggi rurali di interesse storico, commissionata dal Ministero delle politiche agricole, alimentari e forestali (MIPAAF) : "gli strumenti ordinari di conservazione legati al sistema delle aree protette o al vincolo paesaggistico sono inefficaci per conservare il paesaggio agrario " ${ }^{14}$. Forse non serve citare lo straordinario lavoro di Emilio Sereni, Storia del paesaggio agrario italiano, di cui è appena trascorso il cinquantenario (1961-2011): basta scorrere le immagini della recente alluvione di Vernazza nelle Cinque Terre (patrimonio UNESCO) ${ }^{15}$ per constatare ancora una volta quanto sia inscindibile la catena che lega mantenimento delle pratiche agricole, conservazione del paesaggio, salvaguardia della qualità della vita. Anche se in questo caso sarebbe meglio scrivere: conservazione dell'esistenza individuale.

Nonostante l'attuazione degli Osservatori sia stata prevista dalla Convenzione Europea del Paesaggio (datata ormai al 2000); nonostante sia da tempo possibile il confronto con alcuni buoni esempi nazionali (come la ricordata esperienza piemontese e quella, più recente, pugliese) ${ }^{16}$; benché numerosi casi europei - tra i quali spiccano diverse iniziative francesi e quella catalana ${ }^{17}$ - ne dimostrino, almeno sul piano

I3. S. Lagomarsini, "Uso comune e appropriazione metropolitana»; si veda anche M. Quaini, L'ombra del paesaggio, p. 87-100.

I4. Confronta M. Agnoletti, Paesaggi rurali storici, p. 7-9.

15. A Vernazza il crollo dei terrazzamenti abbandonati, fino a tempi molto recenti destinati alla produzione viti-vinicola (prima della definitiva consacrazione turisticomediatica dell'area e dei conseguenti cambiamenti socio-economici), ha causato la frana responsabile di tre vittime umane e della parziale distruzione del nucleo abitato.

I6. URL: http://paesaggio.regione.puglia.it/osservatorio, consultato il 31.01.2012.

17. URL: http://www.catpaisatge.net, consultato il 31.01.2012. 
della produzione di conoscenza, la validità; e infine, pur essendo stato istituito ormai da sei anni un Osservatorio nazionale per la qualità del paesaggio (D.M. 15 marzo 2006 e successive modificazioni e integrazioni) con compiti di promozione di studi e di coordinamento delle iniziative regionali ${ }^{18}$, in Liguria gli Osservatori hanno incontrato il disinteresse più totale, quando non hanno raccolto critiche sulla loro efficacia ${ }^{19}$.

A scala regionale un primo sistematico bilancio dei tentativi di costituzione degli Osservatori, che comunque si sono registrati soprattutto nell'arco degli ultimi tre anni, andrebbe a questo punto effettuato. Ci sono buone ragioni per credere che esso confermerebbe quanto emerso dal rapido monitoraggio effettuato via web. Sembra infatti di assistere, più che all'elaborazione di veri e propri strumenti operativi, all'emergere di poche iniziative che non sembrano andare oltre la dichiarazione d'intenti. Anche quella che appariva la proposta più affidabile, vale a dire l'Osservatorio del paesaggio del Golfo Paradiso, promosso nel 2010 dalla Fondazione Massone di Recco (GE) con l'appoggio della Rete del Golfo Paradiso per il paesaggio e dell'Università di Genova ${ }^{20}$, sembra non avere prodotto studi significativi, né aver elaborato modelli interpretativi e strumenti di monitoraggio efficaci.

Allo stato attuale delle cose bisogna quindi riconoscere che in una regione come la Liguria, che per la straordinaria ricchezza e per la grande

I8. URL: http://www.parc.beniculturali.it/ita/paesaggio/temi/osservatorio.htm, consultati il 31.01.2012. Riporto un breve stralcio tratto da un'interessante critica mossa al modello dell'Osservatorio nazionale da Massimo Quaini (URL: http://www. fondazionemassone.it/public/cmssimple/downloads/Chi\%20siamo\%20cartellina\%20 stampa.pdf, consultato il 31.01.2012): «il Ministero dei Beni Culturali ha approvato la costituzione dell'Osservatorio Nazionale del paesaggio. In una ottica regionale e locale, quale è la nostra, non si vede quale funzione esso possa avere in assenza di Osservatori regionali, che a loro volta per essere coerenti con lo spirito della Convenzione europea devono essere molto vicini ai cittadini. Per questo avanziamo la proposta e la proponiamo alla Regione Liguria e agli enti locali di costituire degli Osservatori di nuova generazione."

19. Alcuni spunti che spingono verso questa tesi si possono rintracciare tra le pagine di un dibattito apparso sul web e datato ottobre 2009, URL: http://preve.blogautore. repubblica.it/2009/10/19/osservatori-per-il-paesaggio/, consultato il 31.01.2012.

20. URL: http://www.fondazionemassone.it/public/cmssimple/index.php? Osservatorio_del_Paesaggio, consultato il 31.01.2012. 
fragilità - che di quella ricchezza è l'altra faccia ${ }^{21}$ - dei suoi paesaggi, in fatto di attivazione di politiche paesistiche dovrebbe essere all'avanguardia, lo strumento "Osservatorio» non ha attecchito. A contrastare iniziative ritenute distruttive dell'integrità di contesti paesaggistici locali sono ancora le singole azioni di gruppi di cittadini, legati ai diversi rami del movimento ambientalista e dell'impegno civile, che trovano nella Rete il più efficace strumento di diffusione delle (eterogenee) informazioni raccolte. Una spinta propositiva «dal basso" che si contrappone al sostanziale, e già in gran parte emerso, disinteresse delle istituzioni. Già nel 2008, tra gli impegni della Regione resi noti al meeting sul paesaggio, evento pubblico purtroppo mai più replicato ${ }^{22}$, nessun riferimento andava agli Osservatori.

La questione del rapporto fra Osservatori e istituzioni merita un, se pur qui breve, approfondimento. A questo proposito la Convenzione Europea non ha dato indicazioni specifiche e le esperienze effettuate in Europa, a cui si è accennato, sono state realizzate con le modalità più varie. Il caso catalano sembra essere quello più istituzionalizzato, essendo la stessa amministrazione pubblica ad avere attivato l'Osservatorio. Un simile esempio in Italia non sembrerebbe avere molto senso. Misurando il problema sul caso ligure, la Regione Liguria, come ogni altra, possiede tutti gli strumenti normativi, da quelli di emanazione nazionale (fra cui, come è noto, non manca neppure il Codice dei Beni Culturali e del Paesaggio) ai propri, per operare nel senso corretto in tema di attivazione di adeguate ed efficaci azioni di monitoraggio, tutela e valorizzazione. Anche se in un Paese come l'Italia non è poi bizzarro che un'istituzione attivi uno strumento utile a promuovere la gestione corretta di ciò che già correttamente essa stessa dovrebbe gestire. La creazione di Osservatori «dal

2I. Se non bastassero i numerosi studi dedicati a questo tema (e mi limito qui a ricordare ancora il volume di M. Quaini, L'ombra del paesaggio), se non bastassero le parole di scrittori come Italo Calvino o Francesco Biamonti (del quale ricordo almeno L'angelo di Avrigue), basta un'escursione in qualsiasi angolo del paesaggio ligure a mostrare come esso sia il risultato di una secolare sfida dell'uomo nei confronti di un territorio difficile, e di come l'allentamento di questa sfida attraverso l'abbandono, lo sfruttamento inconsulto o una malintesa protezione ne decretino la rovina. Su scrittura e pianificazione si veda il recente lavoro di Luisa Rossi, «Fra Zora e Leonia. Geografia, scrittura e pianificazione paesaggistica».

22. L'evento si è svolto tra il 13 e il 15 novembre 2008. Si veda URL: http://www. liguriapaesaggio.it/atti.php?menu=3, consultato il 31.01.2012. 
basso", cioè indipendenti dalle istituzioni, sembra dunque preferibile, in quanto può offrire maggiori garanzie di «democraticità » dello strumento. D'altra parte, come sembra di rilevare dai casi di cronaca, tale modalità può alimentare irrigidimenti e fenomeni di contrapposizione frontale fra istituzioni e cittadini attivi, con il rischio di tradire lo spirito dialogico e partecipativo delle parti che ha ispirato la "carta» degli Osservatori.

Tra le cause - molteplici e analizzabili in maniera molto più puntuale di quanto questo breve contributo consenta - del sostanziale arenarsi delle iniziative degli Osservatori del paesaggio liguri va poi certamente rilevata la mancanza, a monte, di una riflessione teorica specifica riguardante $\mathrm{i}$ metodi d'indagine attivabili, i contenuti e la natura della documentazione prodotta. Riflessione che ormai avrebbe almeno dovuto tradurre il dibattito in corso in un manuale finalizzato a convertire in agevoli schemi, spendibili operativamente, i vari approcci e modelli interpretativi applicati all'analisi del paesaggio. Un testo destinato a fissare una prima griglia interpretativa in grado di fornire una base dialogica comune, utile, nel più lungo periodo, a unire le singole iniziative in una rete regionale.

Chiarire gli obbiettivi e le finalità degli Osservatori significa poi affrontare altri due problemi: quello della conservazione delle conoscenze prodotte (in seguito alle ricerche e alla mediazione dei dibattiti locali) e quello del loro dialogo con i sistemi informativi già attivati dagli enti operanti in campo territoriale, comprese le istituzioni preposte per legge alla conservazione ambientale-paesaggistica (dipartimenti e uffici urbanistici di Regione e Comuni, Soprintendenze per i Beni Architettonici e Paesaggistici, parchi regionali e nazionali, ecc.).

Qualche indicazione - in un senso e nell'altro - è venuta da un recente «scambio» di opinioni seguito alla presentazione di un'iniziativa relativa all'ambito ligure-toscano. Una proposta metodologica (operata sulla base degli esiti di una ricerca applicata, fondata sulle potenzialità delle fonti visive) per la creazione di una base dati funzionale allo studio diacronico del paesaggio all'interno di un Osservatorio.

\section{Osservatorio fotografico del paesaggio e norme figurate}

Il 26 novembre 2011, presso il palazzo mediceo di Seravezza (LU), sono stati presentati gli esiti di una complessa ricerca finalizzata alla realizzazione di un "Osservatorio fotografico del paesaggio" (OF) gravitante 
su una vasta area compresa nei comuni di Seravezza e Stazzema, nel complesso delle Alpi Apuane. Si tratta quindi in un'area montana che le attuali politiche di sviluppo assimilano a molte altre "zone depresse» del Paese, caratterizzata però da una spiccata soggezione alla dicotomia tra sfruttamento minerario (siamo in una delle più pregiate regioni di produzione marmifera a livello mondiale, recentemente legatasi all'industria globale del carbonato di calcio) e protezione ambientale (presenza del Parco Regionale delle Alpi Apuane, ente che potrebbe agevolmente configurarsi come sede dell'OF).

La proposta nasce da una sollecitazione precisa che va ricordata: l'applicazione della metodologia della fotografia ripetuta alla decifrazione diacronica delle evidenze materiali del paesaggio ${ }^{23}$.

L'idea ispiratrice dell'OF prevede che: "con un'inventariazione sistematica e una localizzazione geo-referenziata delle fonti iconografiche storiche di questo territorio e con il loro inserimento metodologico in un più ampio sistema di conoscenza, possa diventare operativamente possibile l'istituzione di un Osservatorio del paesaggio apuano e con esso un percorso virtuoso di manutenzione-riqualificazione» ${ }^{24}$.

Alla presentazione della mostra e del relativo catalogo (dal quale è tratta la citazione precedente), entrambi curati da Orlando Strati, ha fatto da cornice una giornata di dibattito all'interno della quale geografi, urbanisti e amministratori locali ${ }^{25}$ hanno discusso delle metodologie di interpretazione dei palinsesti paesaggistici e delle possibilità di un loro apporto nella messa a punto di strumenti di rappresentazione delle «identità storico-morfologiche dei luoghi ${ }^{26}$.

La conclusione della giornata di studio ha di fatto nuovamente stabilito come priorità imprescindibile per la conservazione dei paesaggi,

23. Per una descrizione più dettagliata e una panoramica storica delle applicazioni italiane si veda C. A. Gemignani, O. Strati, «Verso gli osservatori liguri del paesaggio».

24. O. Strati, Verso un osservatorio apuano del paesaggio, p. 3.

25. Al piccolo convegno, di cui all'URL: http://www.terremedicee.it/eventi_detail. php?idEv=124, consultato il 29.01.2012, hanno partecipato Massimo Quaini (geografo), Roberto Gambino, Fabio Lucchesi, Alberto Magnaghi e Francesco Monacci (urbanisti), Antonio Bartelletti (Direttore del Parco regionale delle Alpi Apuane), Andrea Tenerini (funzionario responsabile del Settore gestione e tutela del territorio del Comune di Seravezza).

26. Si veda D. Poli, «Rappresentazione delle identità storico-morfologiche dei luoghi ». 
della diversità sociale, culturale e biologica ad essi legata e per il contenimento del dissesto ambientale, l'attivazione di efficaci politiche di ripopolamento rurale.

Seguendo lo schema elaborato da Alberto Magnaghi nel suo intervento, lo strumento messo a punto da Strati - che si avvale dell'esperienza delle campagne di ricerca incentrate sulle fonti fotografico-storiche condotte tra 2006 e 2010 nell'ambito del Dottorato di ricerca in "Geografia storica per la valorizzazione del patrimonio storico-ambientale" (Scuola di dottorato "Società, culture, territorio", Università degli Studi di Genova) ${ }^{27}$ - segue un percorso logico che, dall'approccio estetico-percettivo al paesaggio, muove verso una sua interpretazione storico-strutturale e pone come obbiettivo finale il raggiungimento di una sempre più completa conoscenza/coscienza dei luoghi in una prospettiva progettuale e partecipativa. Secondo Magnaghi uno strumento di rappresentazione dei paesaggi come l'OF deve contribuire alla realizzazione di una "normativa figurata": un sistema nel quale ad ogni indirizzo, direttiva o prescrizione, deve corrispondere una rappresentazione grafica (figurazione) «identitaria» della porzione di territorio da sottoporre a normativa ${ }^{28}$.

\section{L'Osservatorio come strumento di valutazione delle politiche locali. Esiti di una discussione}

Il progetto dell'OF apuano ha potuto usufruire di una seconda occasione di confronto nell'ambito delle attività seminariali del dottorato di ricerca in "Geografia storica per la valorizzazione del patrimonio storico-ambientale», durante un incontro tenutosi a Genova il 20 dicembre 2012 presso il Dipartimento di Storia Moderna e Contemporanea. In

27. URL: http://www.dismec.unige.it/ ?section=65, consultato il 29.01.2012. Si veda anche C. A. Gemignani, «Cartografia e fotografia storica per la gestione delle aree protette in Liguria».

28. Di «rappresentazione identitaria» Alberto Magnaghi ha dato la seguente definizione: "una figurazione che, alle diverse scale e con diverse appropriate modalità tecniche, rappresenta i caratteri peculiari del luogo che derivano dalla sintesi di analisi storico-strutturali dei processi di territorializzazione, di analisi morfotipologiche, di analisi ecosistemiche e bioregionali, di analisi estetico percettive" (dall'intervento al Convegno di Seravezza del 16. 11. 2011, cit.). 
quell'occasione storici e geografi presenti ${ }^{29}$ hanno sottolineato un fondamentale nodo critico che tocca non soltanto la metodologia applicata dall'OF - inteso come singolo strumento all'interno di un più complesso sistema di monitoraggio - ma gli scopi e le metodologie d'indagine messi in campo «in generale» dagli Osservatori del paesaggio. Perché la riflessione sulla rappresentazione del paesaggio non si esaurisca in un semplice confronto tra la situazione presente e le rappresentazioni passate, tre sono le azioni che dovrebbero essere attivate secondo una prospettiva di "micro-analisi geografico-storica» ${ }^{30}$ :

1. la ricostruzione filologica delle ragioni che hanno condotto alla produzione della fonte storico-iconografica;

2. la scomposizione delle evidenze visive operata attraverso il confronto;

3. l'analisi dei processi ambientali/territoriali riconosciuti alla base delle trasformazioni e delle continuità rilevate.

Analisi operata con i metodi di incrocio delle fonti adottati dalla geografia storica, dall'ecologia storica e dall'archeologia ambientale ${ }^{31}$. Grazie a questa successione l'aspetto percettivo immediato, che non esaurisce il rapporto conflittuale tra «il paesaggio come immagine» ${ }^{32} \mathrm{e}$

29. Alla giornata hanno partecipato, oltre a Orlando Strati, i membri del collegio docente - tra i quali Diego Moreno, Massimo Quaini e Osvaldo Raggio - e molti tra dottori di ricerca e dottorandi legati ai diversi cicli del Dottorato in Geografia storica per la valorizzazione del patrimonio storico-ambientale.

30. Sul metodo e le finalità si veda R. Cevasco, D. Moreno, «Microanalisi geo-storica o geografia culturale della copertura vegetale?».

3I. R. Cevasco, Memoria verde, p. 69; D. Moreno et al., "L'approccio storicoarcheologico alla copertura vegetale».

32. Da un punto di vista culturale che posto occupa la fotografia storica (di paesaggio o meglio di veduta) in rapporto alla pluralità di approcci sociali alle risorse responsabili delle costruzioni culturali/identitarie locali (recentemente messe in discussione da A. Torre con Luoghi) ? La questione è complessa e merita un lavoro a parte. Se adottiamo l'interpretazione culturale più corrente sul ruolo storico della fotografia nella costruzione di un' «idea nazionale» di paesaggio non possiamo che concordare sul fatto che, lungi dal valorizzare il luogo o dal metterne in evidenza le peculiarità (processi, saperi, conflitti), una semplicistica lettura iconologica la configura piuttosto come un fattore di omologazione: un luogo è paesaggio (fotograficamente parlando) se risponde ad una tradizione iconografica specifica. Su questo tema merita la rilettura di una bibliografia specifica che fa capo, tra gli altri, ad autori come Federico Zeri e Arturo Carlo Quintavalle (si veda, ad esempio, A. C. Quintavalle, «Il territorio della fotografia», p. 3-14). 
il paesaggio come luogo dell'azione, della produzione e dell'abitare (cui ha dedicato molti lavori Luisa Bonesio), può arricchirsi dei dati derivanti dall'identificazione di una più complessa stratificazione di pratiche locali (localizzate) responsabili della costruzione/trasformazione del paesaggio e di più complessi sistemi di attivazione delle risorse ambientali e di contenimento del rischio (geo-idrologico).

Alla base della costituzione di un Osservatorio del paesaggio dovrebbe inoltre porsi la discussione preliminare delle categorie interpretative adottate dalla pianificazione, soprattutto quelle dai confini più incerti («identità dei luoghi», «sguardo quotidiano", ecc.) derivanti dal duplice approccio relazionale - materiale/percettivo - adottato dalla Convenzione Europea ${ }^{33}$. Questa ripresa della discussione dovrebbe fondarsi sulla messa in campo di azioni volte a identificare i metodi di analisi contestuale adottati dalle categorie sociali - attuali e precedenti presenti sul territorio.

A partire da queste sollecitazioni l'utilità degli Osservatori potrebbe venire accresciuta - in chiave, ad esempio, di revisione futura dei Piani se, oltre che come strumenti di coinvolgimento della popolazione nei processi partecipativi, si configurassero pure come strumenti di valutazione critica degli effetti dei sistemi di protezione ambientale adottati in passato. In questa direzione offre notevoli spunti la ricostruzione della difficile gestazione del Piano del Parco Regionale delle Alpi Apuane che, come ricordava Roberto Gambino nella giornata seravezzina, conferisce importanza cruciale al paesaggio - esito sensibile dei processi di territorializzazione - in quanto chiave interpretativa del territorio e strumento al servizio delle strategie di trasformazione rivolte alla sostenibilità. Le conclusioni proposte da Gambino sono ben sintetizzate da alcune brevi considerazioni che meritano, in conclusione, di essere riportate come elementi base della logica che dovrebbe animare gli Osservatori, se si deciderà di puntare operativamente sulla loro fondazione:

[...] nella logica del Piano, l'interpretazione strutturale del territorio svolge l'indispensabile funzione di rappresentare gli argini immodificabili entro i quali possono prendere forma le scelte strategiche di sviluppo sostenibile, con la flessibilità resa opportuna dalla complessità, dall'incertezza e dalla scarsa prevedibilità delle dinamiche territoriali ed ambientali. Flessibilità che obbliga ad uscire

33. Si vedano le riflessioni di M. Quaini in "Il paesaggio». 
dalle tradizionali gabbie vincolistiche per avviare piuttosto processi concertativi che coinvolgano i diversi soggetti del governo territoriale e i diversi portatori di interessi [...]. Il quadro strategico comporta quindi il confronto dinamico di scenari alternativi [...] il rapporto col contesto non riguarda soltanto le relazioni spaziali, bensì anche quelle socioculturali, come è emerso con chiarezza nell'esperienza del Forum e dei processi di consultazione, che hanno coinvolto in modo significativo le popolazioni e gli amministratori locali e il mondo delle associazioni [...].

A quindici anni di distanza dalle proposte preliminari, a dieci dalla proposta definitiva, le indicazioni del Piano in queste tre direzioni sembrano ancora largamente disattese, mentre i processi reali hanno spesso rovinosamente smentito gli obiettivi strategici e reso più difficile la conservazione di un territorio ricco di uno spettacolare patrimonio naturale e culturale. La realizzazione dell'Osservatorio Apuano potrà fare un passo significativo a questo fine, soprattutto se non si chiuderà nella difesa delle "eccellenze» ma saprà affrontare il ben più rischioso compito della valorizzazione autentica dell'intero territorio ${ }^{34}$.

\section{Carlo A. Gemignani}

Università di Trento

34. I testi sono tratti dalle slides della presentazione mostrata durante il convegno, gentilmente messa a disposizione dal Prof. Gambino. 


\section{BIBLIOGRAFIA}

Agnoletti, Mauro (a cura di), Paesaggi rurali storici. Per un catalogo nazionale, Roma/Bari, Laterza, 2010.

Baldeschi, Paolo, Paesaggio e territorio, Firenze, Le lettere, 2011.

Cevasco, Roberta, Memoria verde. Nuovi spazi per la geografia, Reggio Emilia, Diabasis, 2007.

Cevasco, Roberta, Moreno, Diego, «Microanalisi geo-storica o geografia culturale della copertura vegetale? Sull'eredità ambientale dei "paesaggi culturali" ", Trame nello spazio. Quaderni di geografia storica e quantitativa, 3 (2007), p. 83-101.

Garibaldi, Andrea et al., La Colata. Il partito del cemento che sta cancellando l'Italia e il suo futuro, a cura di Ferruccio Sansa, Milano, Chiarelettere, 2010.

Gemignani, Carlo Alberto, "Cartografia e fotografia storica per la gestione delle aree protette in Liguria", Semestrale di Studi e Ricerche in Geografia, 2 (2010), p. 149-159.

Gemignani, Carlo Alberto, Strati, Orlando, "Verso gli osservatori liguri del paesaggio. Una campagna di fotografia ripetuta nel sito UNESCO "Cinque Terre, Portovenere e le isole" (La Spezia)", Rivista Geografica Italiana, 118 (2011), p. 525-557.

Lagomarsini, Sandro, "Uso comune e appropriazione metropolitana: due modelli di utilizzo del territorio in Val di Vara", Memorie della Accademia Lunigianese di Scienze "Giovanni Capellini", 60 (2000), p. 75-89.

Moreno, Diego et al., «L'approccio storico-archeologico alla copertura vegetale: il contributo dell'archeologia ambientale e dell'ecologia storica", in La biologia vegetale per $i$ beni culturali. II. Conoscenza e valorizzazione, a cura di Giulia Caneva, Firenze, Nardini, 2005, p. 463-498.

PolI, Daniela, "Rappresentazione delle identità storico-morfologiche dei luoghi", in Rappresentare i luoghi. Metodi e tecniche, a cura di Alberto Magnaghi, Firenze, Alinea, 2011, p. 215-285. 
Preve, Marco, Sansa, Ferruccio, Il partito del cemento. Politici, imprenditori, banchieri. La nuova speculazione edilizia, Milano, Chiarelettere, 2008.

Quaini, Massimo, L'ombra del paesaggio. L'orizzonte di un'utopia conviviale, Reggio Emilia, Diabasis, 2005.

—, "Il paesaggio: un percorso tra mercificazione e convivialità ", in Paesaggio: l'anima dei luoghi, a cura di Luisa Bonesio, Luca Micotti, Reggio Emilia, Diabasis, 2008, p. 27-48.

Quintavalle, Arturo Carlo, "Il territorio della fotografia", in Enciclopedia pratica per fotografare, Milano, Fabbri, 1979, p. 3-32.

Rossi, Luisa, "Fra Zora e Leonia. Geografia, scrittura e pianificazione paesaggistica", in Paesaggio ligure e paesaggi interiori nella poesia di Eugenio Montale, a cura di Paola Polito, Antonio Zollino, Firenze, Olschki, 2011, p. 175-189.

Settis, Salvatore, Paesaggio, Costituzione, cemento. La battaglia per l'ambiente contro il degrado civile, Torino, Einaudi, 2010.

Strati, Orlando, Verso un osservatorio apuano del paesaggio, Firenze, Alinea, 2011.

Torre, Angelo, Luoghi. La produzione di località in età moderna e contemporanea, Roma, Donzelli, 2011.

Vaccarezza, Claudia, Paesaggi rurali. Tra storia delle risorse e morfologia sociale, Scuola di Dottorato di Ricerca in Società, culture, territorio - Ciclo XXIII, Geografia storica per la valorizzazione del patrimonio storico-ambientale, Università degli Studi di Genova, Genova, 2011. 